\title{
SPARSITY-BASED DECONVOLUTION OF LOW-DOSE BRAIN PERFUSION CT IN SUBARACHNOID HEMORRHAGE PATIENTS
}

\author{
Ruogu Fang ${ }^{1}$, Tsuhan Chen ${ }^{1}$, Pina C. Sanelli ${ }^{2}$ \\ ${ }^{1}$ Department of Electrical and Computer Engineering, Cornell University, Ithaca, NY, USA \\ ${ }^{2}$ Department of Radiology, Weill Cornell Medical College, NY, NY, USA
}

\begin{abstract}
Functional imaging serves as an important supplement to anatomical imaging modalities such as MR and CT in modern health care. In perfusion CT (CTP), hemodynamic parameters are derived from the tracking of the first-pass of the contrast bolus entering a tissue region of interest. In practice, however, the post-processed parametric maps tend to be noisy, especially in low-dose CTP, in part due to the noisy contrast enhancement profile and oscillatory nature of results generated by current computational methods. In this paper, we propose a sparsity-based perfusion parameter deconvolution approach that consists of a non-linear processing based on sparsity prior in terms of residue function dictionaries. Our simulated results from numericaldata and experiments in aneurysmal subarachnoid hemorrhage patients with clinical vasospasm show that the algorithm improves the quality and reduces the noise of the perfusion parametric maps in low-dose CTP, compared to state-of-the-art methods.
\end{abstract}

Index Terms - sparse representation, residue function, truncated singular value decomposition (TSVD), perfusion computed tomography (CTP), aneurysmal subarachnoid hemorrhage

\section{INTRODUCTION}

Low-dose computed tomography (CT) has attracted increasing attention in recent years, especially in perfusion CT (CTP) imaging, which has higher radiation dose due to its cine scanning technique resulting in repetitive imaging on a focused region of the body. CTP provides functional information complimentary to the standard imaging modalities in modern medicine to characterize hypoperfused tissue. CTP has been useful in quantitatively and qualitatively assessing hemodynamic parameters, including cerebral blood flow (CBF), cerebral blood volume (CBV) and mean transit time (MTT). These quantities can provide insights into, for instance, the degree to which hypoperfused brain tissue is at risk of infarction and irreversible recovery. However, several recent reports of radiation overexposure has raised significant concerns to patients and radiologists regarding its usage. The method to obtain high- quality post-processed parametric maps from a low-dose scan remains a challenge for this technique.

Conventionally, truncated singular value decomposition (TSVD) is used to estimate the impulse residue function (IRF) via deconvolution $[1,2]$, which has been shown to have an oscillatory nature [3]. In case of low-dose CTP, the increased noise in the CT data combined with the potentially oscillatory TSVD creates parameter maps that are very noisy. Most state-of-the-art noise reduction approaches for low-dose CTP are based on preprocessing of CT data before applying algorithms to determine perfusion parametric maps. Numerous works have been proposed and have successfully improved the quality of CBF maps, including bilateral filtering, non-local mean, nonlinear diffusion filter, and wavelet-based methods [4-7]. However, these works do not take the convolution flow model of perfusion CT into consideration. Therefore we sought to develop a new deconvolution method that can learn from the high-dose data to improve the quality of low-dose perfusion parametric maps.

In this paper, our goal is to improve low-dose CTP parametric map computation. The approach is to impose a sparsity prior on low-dose dynamic 3D CT data, in terms of a dictionary $\mathrm{D}$ obtained from high-dose CTPresidue function. A linear transform from the dictionary is performance to restore the high quality perfusion parametric maps based on the property that residue functions in lowdose CTP can be well approximated by a linear combination of just a few columns from the high-dose residue functions in $\mathrm{D}$. The transform is adapted to the observed low-dose dynamic CT data with convolution flow model and the data statistics from the training set. Sparsity-based method has been applied on sonogram and CT data before perfusion maps computation, but to the best of our knowledge, its direct application on improving perfusion parametric deconvolution in CTP has not been explored yet.

Our major contributions in this work are two-fold: First, we propose a novel framework for deconvolution of perfusion CT data using sparsity prior in terms of high-dose residue function dictionary. Second, the algorithm improves the low-dose CTP quantification by learning from the data and computing the perfusion parameters "on the fly", instead of a pre-assume any explicit noise model. 


\section{APPROACH}

\subsection{Perfusion Parameter Estimation}

In a dynamic contrast enhanced imaging study, $C_{v}(t)$ represents the venous time-enhancement curve and $C_{a}(t)$ represents the artery input. $C B F$ is the cerebral blood flow and $R(t)$ is the tissue impulse residue function, which measures the mass of contrast media remaining in the given vascular network over time. At the beginning $(t=0)$, a unit of contrast material $(R(0)=1)$ is injected as a bolus at an arterial input, and after a finite duration, as contrast material begins to leave the vascular network, $R$ drops to zero. The amount of contrast in a region is characterized by

$$
C_{v}(t)=C B F \cdot \int_{0}^{t} C_{a}(\tau) R(t-\tau) d \tau
$$

\subsection{Sparsity Residue Representation (SRR)}

We propose a deconvolution method aiming to achieve robust estimation of perfusion parameters in low-dose/noisy CTP data. Instead of assuming any parametric model of perfusion statistics, we propose to incorporate residue prior on-the-fly through sparse representation. More specifically, we have the following sparsity observation: Given a large repository of residue functions computed from high-dose CTP data of an organ, the residue functions in the low-dose CTP data of the same organ can be approximated by a linear combination of a sparse set of instances in the residue function repository.

We define a spatial-temporal patch as a $3 \mathrm{D}$ patch along with its temporal data. Let $C_{v}(x, y, z, t) \in \mathbb{R}^{N^{3} \times T}$ represent the input tracer concentration in the tissue of the voxel $[x, y, z]^{T}$ from a spatial-temporal patch of size $\mathrm{N} \times \mathrm{N} \times \mathrm{N}$ pixels and $T$ time points, and $R(x, y, z, t) \in \mathbb{R}^{N^{3} \times T}$ represent the remaining tracer concentration of the voxel $[x, y, z]^{T}$ at a given time point $t$, where $x, y$ and $z$ are the respective row, column and slice coordinates of the data. The approximation of patch-wise residue functions $R$ is then formulated as an optimization problem:

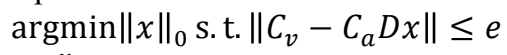

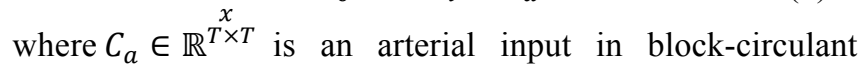
version, as shown in Section 2.1. $D \in \mathbb{R}^{T \times N^{3} \times K}$ is a dictionary which is obtained from high-dose CTP data, which is comprised of 4D elements of the residue functions in a voxel of interest (VOI). The residue functions of the spatial-temporal patch $R$ is approximated by $D x$, where $x \in \mathbb{R}^{K}$ denotes the coefficient/weights of linear combination to encode the observed residue function from low-dose CTP data in terms of D. The $L_{0}$ norm $\|x\|_{0}$ is the number of non-zero elements in $x$ and ensures a sparse set of residue function instances can be used to approximate the input tracer concentration, which prevents the overfitting to error from missing/misleading tracer concentration. $\varepsilon$ is the prescribed error tolerance of representation error. The constraint ensures fidelity to the observation.
(2) is an NP hard problem owing to the non-convent $L_{0}$ norm. Thanks to the recent proof of the sparse representation theorem [8], $\mathrm{L}_{1}$ norm relaxation can be employed to make the problem convex while still preserving the sparsity property.

$$
\underset{x}{\operatorname{argmin}}\left\|C_{v}-C_{a} D x\right\|_{2}^{2}+\mu\|x\|_{1}
$$

where $\mu$ is the sparsity parameter that controls the number of non-zeros elements in $x$. Since (3) now becomes a typical linear inverse problem, it can be solved using existing solvers[9]. $D x$ is then computed as a refined version of the residue functions of the input patch-wise tracer concentration, which imposes the residue priors on-the-fly. Here we use block-circulant version of $C_{a}$ matrix in our implementation [2].

\section{EXPERIMENTS AND RESULTS}

In this section, we first show our results from simulationbased experiments, followed by results from our clinical evaluations.

\subsection{Simulations}

The AIF is simulated using a gamma-variant function:

$$
C_{a}(t)=\left\{\begin{array}{cc}
0 & t \leq t_{0} \\
a\left(t-t_{0}\right)^{b} e^{-\left(t-t_{0}\right) / c} & t>t_{0}
\end{array}\right.
$$

where $t_{0}$ is bolus arrival time to any given region.

Transport function $h(t ; \theta)$, with $\theta$ as the model parameter, is simulated using the family of gamma distributions

$$
h(t ; \alpha, \beta)=\frac{1}{\beta^{\alpha} \Gamma(\alpha)} t^{\alpha-1} e^{-t / \beta} \quad \alpha, \beta>0
$$

Here $\beta=B V /(\alpha \cdot B F)$ to ensure the central volume theorem. The residue function can be derived from the transport function $h(t)$ by

$$
R(t)=1-\int_{0}^{t} h(\tau) d \tau
$$

The tissue enhancement curve within the given VOI is generated using

$$
C_{v}(t)=C B F \cdot \int_{0}^{t} C_{a}(\tau) R(t-\tau) d \tau
$$

We set $a=1, b=3, c=1.5, t_{0}=0$ to generate the AIF. $\mathrm{CBV}=4 \mathrm{~mL} / 100 \mathrm{~g}$, and $\mathrm{CBF}$ values varies from 20 to 80 $\mathrm{mL} / 100 \mathrm{~g} / \mathrm{min}$ in $10 \mathrm{~mL} / 100 \mathrm{~g} / \mathrm{min}$ increments. Correspondingly, $\mathrm{MTT}=\mathrm{CBV} / \mathrm{CBF}$ and ranges from 3 to 12 seconds. For residue function, $\alpha=10,(\beta=M T T / \alpha)$. Gaussian noise $\varepsilon \sim \mathrm{N}\left(0, \sigma^{2}\right)$ is then added to the noise-free tissue time-enhancement curve generated using (7). Peak signal-to-noise ratio (PSNR) is calculated by dividing the peak value of the tissue time-enhancement signal by the noise standard deviationo.s

$$
P S N R=10 \log _{10} \frac{C_{\max }^{2}}{\sigma^{2}}
$$

where $C_{\max }$ is the peak value of the tissue timeenhancement signal. The dictionary $D$ is simulated using residue functions generated at various $\mathrm{CBF}$ values from 1 to $100 \mathrm{~mL} / 100 \mathrm{~g} / \mathrm{min}$, with CBV constant at $4 \mathrm{~mL} / 100 \mathrm{~g}$. 
(1a)

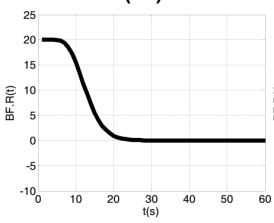

(2a)

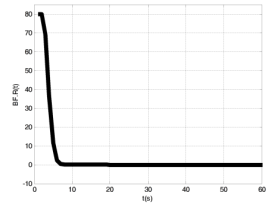

(1b)

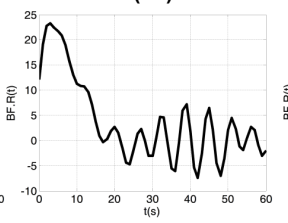

(2b)

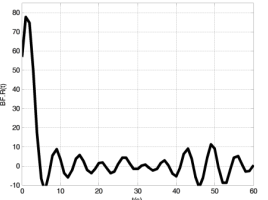

(1c)

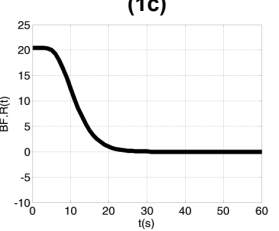

(2c)

Fig. 1. Recovery of residue functions by cTSVD and sparse residue representation methods. The parameters used for the simulation is $\mathrm{BV}=4 \mathrm{ml} / 100 \mathrm{~g}$. In the first row, $\mathrm{PSNR}=20$ and $\mathrm{BF}=20 \mathrm{ml} / 100$ $\mathrm{g} / \mathrm{min}$; in the second row, $\mathrm{PSNR}=40$ and $\mathrm{BF}=80 \mathrm{ml} / 100 \mathrm{~g} / \mathrm{min}$. Column (a): True residue function. Column (b): Restored residue function by cTSVD. Column (c): Restored residue function by sparse residue representation.

\subsection{Simulation Results}

Residue Function Recovery: The residue function recovered by cTSVD and SR deconvolution methods are shown in Fig. 1. Under both circumstances of PSNR $=20$ and $\mathrm{PSNR}=40$, the residue functions in Fig. 1 (1c) and (2c)are in agreement with the true residue best. On the other hand, the curves in Fig. 1 (1b) (2b) for cTSVD show relatively large baseline oscillations and less accurate peak values.

CBF Estimation: From the recovered residue function, $\mathrm{CBF}$ can be estimated at $\mathrm{t}=0$. Now we examine the accuracy of $\mathrm{CBF}$ estimates at various noise levels. We use single voxel to compare the performance of cTSVD and sparse residue representation. The comparison is carried out at different values of $\mathrm{CBF}$ and at both low and high noise levels. The simulations are repeated 25 times with random noise realizations.

Fig. 2 shows the CBF values as estimated by cTSVD and sparse residue representation, for two different noise levels. SR can be visually observed to be more accurate and robust. To further summarize Fig. 2, we compute the mean squared error (MSE) $\varepsilon$ as defined by

$$
\varepsilon=\frac{1}{n} \sum_{i=1}^{n}\left(f_{i}-\widehat{f}_{l}\right)^{2}
$$

where $f_{i}$ and $\widehat{f}_{l}, i=1,2, \ldots, n$, are the true and estimated CBF values, respectively. The MSEs of cTSVD and sparse residue representation are 47.1007 and 2.4974 , respectively, at $\mathrm{PSNR}=10$, and 9.1072 and 0.1536 at $\mathrm{PSNR}=40$.

\subsection{Clinical Applications}

In this section, we describe the results from comparing our approach with cTSVD on four subjects - two with ischemia related to vasospasm. The presence and location of the perfusion deficits were identified by board-certified neuroradiologists.
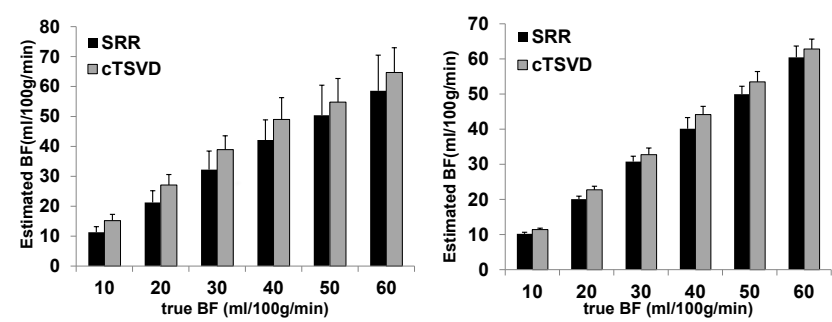

Fig. 2. Comparison of the accuracy in estimating BF by cTSVD and sparse residue representation methods for different $\mathrm{BF}$ values. (a) $\mathrm{PSNR}=10, \mathrm{MSE}=2.4974$ for sparse residue representation and $\mathrm{MSE}=47.1007$ for $\mathrm{cTSVD}$; (b) $\mathrm{PSNR}=40, \mathrm{MSE}=0.1536$ for sparse residue representation and MSE $=9.1072$ for $\mathrm{cTSVD}$.

There is a standard scanning protocol for CTP using GE Lightspeed or Pro-16 scanners (General Electric Medical Systems, Milwaukee, WI) with cine 4i scanning mode and 45 second acquisition at 1 rotation per second using $80 \mathrm{kVp}$ and $190 \mathrm{~mA}$.

To estimate the perfusion parametric maps in high-dose CTP, we use cTSVD to compute the residue functions in high-dose data and build a dictionary $\mathrm{D}$ by randomly choosing 10,000 residue functions from the training data using 3 independent patient cases.

For cTSVD, a threshold of $6 \%$ of the maximum singular value is used, in accordance with parameter tuning in our experiments. The regularization parameter for sparse residue representation is set $\lambda=0.1$. Gaussian noise was added to each dataset to simulate low-dose CTP data with PSNR $=20$. Processing is performed on a computer with Intel Core 2 Duo CPU, E8400@3.00GHz and 3.00GHz, 3GB of RAM. It takes approximately 300 seconds to process a ROI of $80 \times 60$ voxels by sparse residue representation method, and approximately 0.2 seconds by the cTSVD method.

CBF maps were calculated for each dataset at both high dose and low dose using cTSVD and sparse residue representation deconvolution. First, we evaluated the qualitative appearanceof the estimated CBF maps. As shown in Fig 3visible noise in the estimated CBF maps of the two patients are greatly reduced by our approach. The low CBF caused by ischemia related vasospasm is more evident due to improved spatial resolution and contrast resolution. This conclusion can be further validated by calculating and comparing the contrast to noise ratio and standard deviation of CBF values over the regions of interest (ROI) in each map which are sampling the anterior cerebral artery territory. Table 1 shows that the variations (standard deviations) of the $\mathrm{CBF}$ values estimated via our sparse representation method are highly improved compared to those estimated via TSVD on simulated low dose dataset. Meanwhile, since we have the ground truth data of CBF maps at high dose of $190 \mathrm{~mA}$, we also compute the mean-square-error (MSE) of the estimated CBF maps using sparse residue representation and TSVD with the high dose CBF maps. 


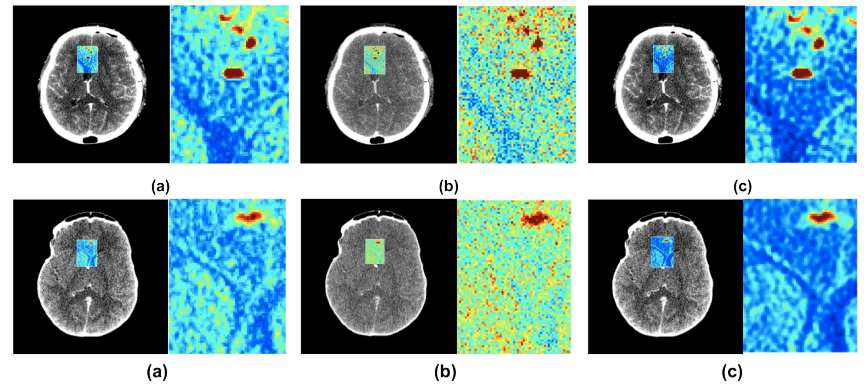

Fig. 3. BF maps and zoomed-in regions of a vasospasm patient (above row) and normal patient (below row) using (a) high-dose TSVD (b) low-dose TSVD and (c) low-dose sparse residue representation.

\begin{tabular}{|c|c|c|c|c|}
\hline \multirow{2}{*}{ Subjects } & \multicolumn{2}{|c|}{ Variations } & \multicolumn{2}{c|}{ MSE } \\
\cline { 2 - 5 } & SRR & TSVD & SRR & TSVD \\
\hline 1 & 23.96 & 38.06 & 179.38 & 745.68 \\
\hline 2 & 26.68 & 46.40 & 263.42 & 545.38 \\
\hline 3 & 28.23 & 33.99 & 201.38 & 865.492 \\
\hline 4 & 18.46 & 34.29 & 229.45 & 715.59 \\
\hline
\end{tabular}

Table 1. BF variations $(\mathrm{ml} / 100 \mathrm{~g} / \mathrm{min})$ and mean square error (MSE) over certain ROIs estimated by sparse residue representation and TSVD.

In addition, by aggregating all voxels (within the ROI) from the two normal patient data sets into a single "normal" group, and two ischemia patient data sets into an "abnormal" group, we have 2 clusters of voxels - one containing $\mathrm{n}_{1}$ samples of CBF values from normal anterior cerebral artery territory, and other containing $\mathrm{n}_{2}$ samples of $\mathrm{CBF}$ values from vasospasm voxels in the anterior cerebral artery territory. In our case, $\mathrm{n}_{1}=2000$ and $\mathrm{n}_{2}=2000$. To quantify the separability between normal and ischemic CBF values, we define the distance between these two clusters as:

$$
d=\frac{\mu_{1}-\mu_{2}}{\sqrt{\sigma_{1}^{2} / n_{1}+\sigma_{2}^{2} / n_{2}}}
$$

where $\mu_{1}, \mu_{2}$ are the means, and $\sigma_{1}, \sigma_{2}$ are the standard deviations of $\mathrm{CBF}$ in the normal and ischemic clusters respectively. We expect our sparse-residue representation deconvolution algorithm to produce larger distance $d$ as defined in Eq. (10), that is, to more definitely differentiate between normal and ischemic patients. Fig. 4 show scatter plots of normal vs. ischemic clusters. It is apparent that the two clusters are more separable in data processed via sparse residue representation deconvolution than TSVD.

\section{CONCLUSION}

In conclusion, we have presented a sparse residue representation deconvolution method to calculate CTP parametric maps for low dose CTP. The experimental results show that by learning a dictionary ofresidue functions from the high-dose CTP data, our method outperforms TSVD inboth reducing the variance within local homogeneous regions and enhancing the color contrast of $\mathrm{CBF}$ valuesbetween normal and ischemic patients, potentially improving the differentiation of ischemia related to vasospasm in these patients.

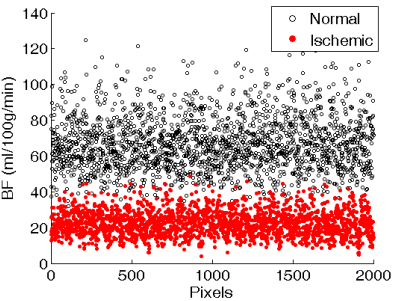

(a)

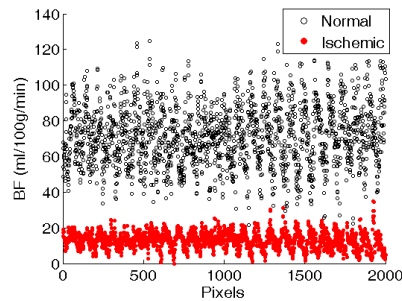

(b)
Fig. 4. (a) Two clusters of normal vs. abnormal generated by TSVD method. The distance $d$ between two clusters is 118.08. (b) Two clusters of normal vs. abnormal generated by our sparse residue representation method. The distance $\mathrm{d}$ between two clusters is 148.57 .

In our proposed method, when the learning procedure is accomplished in an off-line training, the resulting algorithm can benefit from both low computational cost of noniterative data processing and improved performance due to the learning. More importantly, this adaptive deconvolution method can be supplemented with all existing preprocessing approaches on CT data [4-7] and improved deconvolution algorithms to compute perfusion parameters [10].

\section{REFERENCES}

[1] L. Ostergaard, R.M. Weisskoff, D.A. Chesler, C. Gyldensted, and B.R. Rosen, "High resolution measurement of cerebral blood flow using intravascular tracer bolus passages. Part 1: Mathematical approach and statistical analysis," Mag. Res. Med., vol. 36, pp. 715-725, 1996.

[2] O. Wu, L. Ostergaard, R.M. Weisskoff, T. Benner, B.R. Rosen, and A.G. Sorensen, "Tracer arrival timing-insensitive technique for estimating flow in MR perfusion-weighted imaging using singular value decomposition with a block-circulant deconvolution matrix," Mag. Res. Med. Vol. 50, pp. 164-174, 2003.

[3] K. Mouridsen, K. Friston, N. Hjort, L. Gyldensted, L. Ostergaard, and S. Kiebel, "Bayesian estimation of cerebral perfusion using a physiological model of microvasculature," NeuroImage, vol. 33, pp. 570-579, 2006.

[4] Y. Tao, S. T. Brunner, J. Tang, H. A. Rowley, G. Chen. "Radiation dose reduction in computed tomography (CT) using a novel implementation of wavelet denoising in low tube current acquisitions," SPIE Medical Imaging 2011.

[5] H. Watanabe, D. Tsai, Y. Lee, E. Matsuyama. K. Kojima. "Radiation dose reduction in digital radiography using wavelet-based image processing methods," SPIE Medical Imaging 2011.

[6] A.M. Mendrik, E.J. Vonken, B. van Ginneken, H.W. de Jong, A. Riordan, T. van Seeters, E.J. Smit, M.A. Viergever, M. Prokop. "TIP bilateral noise reduction in 4D CT perfusion scans produces high-quality cerebral blood flow maps," Phys. Med. Bio. vol. 56, pp. 3857-3872, 2011.

[7] N. Saito, K. Kohsuke, S. Tsukasa, U. Masahito, K. Kazuhiro, M. Kichiko, S. Shigenhito. "Realization of reliable cerebral-blood-flow maps from low-dose CT perfusion images by statistical noise reduction using non-linear diffusion filter," Radiological physics and technology, vol. 1, no. 1, pp. 62-74. 2008.

[8] Donoho, D., "For most large undetermined systems of equations, the minimal 11-norm near-solution approximates the sparest near-solution," Communications on Pure and Applied Mathematics 59(7), 907-934, 2007. [9] J. Liu, S. Ji, and J. Ye, "SLEP: Sparse Learning with Efficient Projections. Arizona State University,"

2009. http://www.public.asu.edu/ jye02/Software/SLEP.

[10] L. He, B. Orten, S. Do, W.C. Karl, A. Kambadakone, D.V. Sahani, H. Pien, "A spatio-temporal deconvolution method to improve perfusion CT quantification," IEEE Transactions on Medical Imaging, vol. 29, no. 5, pp. 1182-1191, 2010. 